\title{
Subjective Assessment of Adaptive Media Playout for Video Streaming
}

\author{
Pablo Pérez, Narciso García, and Álvaro Villegas
}

\begin{abstract}
Adaptive Media Playout (AMP), the adaptive modification of media playback speed, has been previously proposed as a technique to modify the playout delay of a user with respect to other users or with real time, thus having a crucial role in social TV and live video streaming. In this paper, we study the subjective impact of the playout rate change, which had received very limited attention in the past. In particular, we analyze the effect of the subject and the source content in the subjective opinion.

We have found that most of the previous works underestimated the impact of AMP in perceived quality, and that the speed change rate should not be modified beyond $10 \%$ from the original, as a general rule. We have also developed a user scoring model which takes into account the variability between users and sources. Our results can help developing cost models for playout control systems based on AMP, as well as provide some insights to the analysis of other types of subjective assessment tests.

Index Terms-adaptive media playout, subjective assessment, subject model
\end{abstract}

\section{INTRODUCTION}

A well-known restriction of live video streaming is that the end-to-end delay, and therefore the reception buffer capacity (in seconds), must remain constant for the whole duration of the session [1]. If the buffer is too small, there will be risks of underrun, which impairs Quality of Experience (QoE) dramatically [2]. On the other hand, if the buffer is too large, the end-to-end delay will also be high, which is undesirable too [3]. In live streaming using adaptive bitrate (ABR) over http (e.g. MPEG-DASH), this trade-off is normally broken in favor of a large buffer, typically in the range of tens of seconds, resulting in better resilience to bandwidth changes, at the cost of a latency which is much higher than the one provided by digital television broadcasting technologies [4].

So far this problem has received limited attention, since most critical live events (typically sport games) are traditionally being served through broadcasting technologies, and ABR streams are usually a secondary option. However, this situation is changing as some of the biggest streaming platforms (Amazon, Facebook, YouTube) are starting to offer live television streams. For those platforms, being able to control the end-toend delay in a more flexible way would make it possible to improve their end-to-end QoE (e.g. by providing lower latency for the contents, or sections of content, which are more time sensitive). Additionally, there are some scenarios where several clients need to playout their streams synchronously, in what it is called Inter-Destination Media Synchronization (IDMS) [5]. For them, managing the end-to-end delay would also allow for re-sychronization of out-of-sync streams.

Fortunately, there is a way to break the constant delay restriction: modifying the playout speed in the client so that the buffer is consumed at a different speed than it is filled. Of course, to avoid that the buffer empties or that the latency increases indefinitely, this playout speed variation must be adaptive on its own. The ability to change the playout speed to help manage the client buffer is called Adaptive Media Playout (AMP) [6]. Its concept is relatively straightforward: the buffer size is modified by changing the playout speed at the client side. Video playout speed changes are achieved by repeating or dropping frames where needed. Audio speed changes uses Waveform Similarity Overlapp-Add (WSOLA) to modify the playout speed without modifying the signal pitch [7]. Since its proposal at the beginning of the 2000s, AMP has been proposed for buffer control in situations where the instant channel throughput drops below the content bitrate [8]-[10], to handle segments of different bitrate over a constant-bitrate channel [11], or to perform IDMS [12], [13].

Most of the studies on AMP have evaluated its capacities of buffer management to avoid underruns under latency constraints [14]. In those studies, the effect of the speed modification in the quality is considered linear [13] or quadratic [14] to the playout rate or to the frame period variation [10], sometimes including an additional factor such as the motion intensity [15]. However, none of those studies seem to have been derived from actual subjective experiments, beyond Kalman et al. reporting that "informal tests have shown that playout speed variations of up to $25 \%$ are often not noticeable, and depending on the content, rate variations up to $50 \%$ are sometimes acceptable" [8].

Some subjective studies have appeared more recently. Rainer and Timmerer used a crowdsourcing subjective test to build a QoE model for AMP [16]. Among other results, they showed that the impact on $\mathrm{QoE}$ is higher when decreasing speed by a $1 / G$ factor than when increasing it by a $G$ factor. However, the test used a single source content both to train 
TABLE I

SOURCE CONTENTS

\begin{tabular}{|l|c|c|l|}
\hline ID & Type & Name & Description \\
\hline 01 & Sports & Sprint & Bolt wins 100m final run at Olympics \\
02 & Sports & Goal & Real Madrid scores in football match \\
03 & Sports & NBA & Kobe Bryant scores at NBA \\
04 & Music & Radetzky & Radetzky March at New Year Concert \\
05 & Music & Queen & We Are The Champions music video \\
06 & Music & Sobral & Salvador Sobral at Eurovision final \\
07 & Speech & PM & Prime Minister speech at Parliament \\
08 & Speech & Show & Magic trick at TV show 'El Hormiguero' \\
09 & Speech & News & Matias Prats introducing news \\
10 & Fiction & Crime & Parody of crime scene show \\
11 & Fiction & Tiempo & El Ministerio del Tiempo TV show \\
12 & Fiction & Galaxy & Guardians of the Galaxy animation \\
13 & Action & Rogue & Rogue One space battle scene \\
14 & Action & Clone & Clone Wars animation: light saber fight \\
15 & Action & Wall-E & Wall-E animation: robots \\
\hline
\end{tabular}

and validate the model, and therefore its results might not be generalizable to other contents. Mu et al. tested six different sources, as well as five speed gains $(\times 1.1$ to $\times 2)$ and four durations (1-8 seconds) of AMP application [17]. They also developed a model for AMP quality impact depending on gain and time, which shows that the effect of duration in quality starts to saturate at about 6 seconds. However, they only covered speed increase (not decrease), and the impact of the source content in the QoE was not addressed.

In this paper, we try to overcome some of the limitations of the previous studies and, in particular, the influence of the content source and the characterization of the users. We have designed an experiment with a careful selection of the sources, which has been evaluated by a high number of users, described in Section II. Results have been evaluated with standard statistical tools (Section III), and a user model has been developed to account for the contribution of source and user to the scores (Section IV). Section V discusses the results, and Section VI presents the conclusions.

\section{EXPERIMENTAL DESIGN}

One of our main concerns when designing the experiment was getting a set of contents and impairments that does not underestimate the effect of AMP in QoE. As there are very few previous experiments on the topic, it is particularly relevant to avoid overfitting the results to a particular set of sources.

With this purpose, we have selected a set of 15 different content sources which are "demanding, but not unduly so" [18], summarized in Table I. Sources belong to one of these categories: Sports, Music, Speech (non-fiction, person speaking to camera), Fiction (fiction contents, people speaking), Action (fiction contents, action scenes). Sources 12, 14 and 15 come from animation/CGI content. Sequences are selected to have a recognizable time structure, so that any distortion in playout speed is easy to identify. They are cuts from very popular sports, music pieces, TV shows and movies, available on the internet (YouTube, rtve.es, etc.). Speakers in the Speech category are well-known personalities, whose voice and speech dynamics are quite recognizable by an average user.

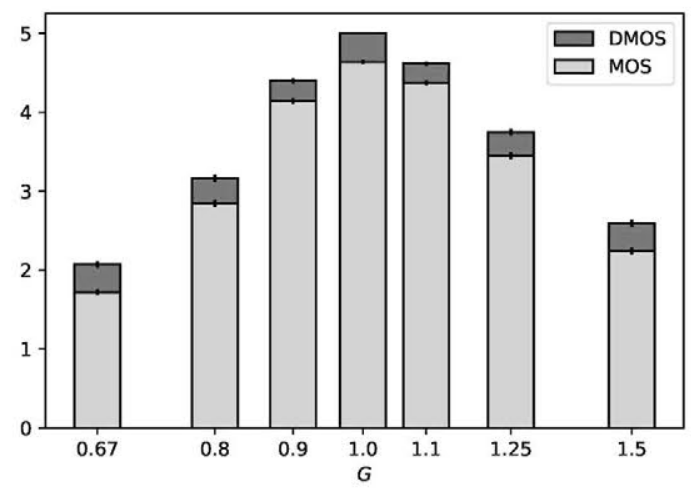

Fig. 1. MOS and DMOS for each speed gain $G$.

For the test, one 10 second sequence was cut from each one of the original sources. Sequences had a resolution of $1280 \times$ 720 and 50 frames per second, as well as stereo audio, with enough bitrate to present no visible coding artifact. In each of the sequences, a playout rate variation was introduced: after the first 4 seconds, the playout speed was changed to $G$ times the original. Values of $G$ were $0.67,0.8,0.9,1.0$ (hidden reference), $1.1,1.25$ and 1.5 . We decided to introduce the variation in the middle of the sequence to capture the response of the user to a speed change, similarly to what was done by Mu et al. [17]. The duration of the impairment was then about 6 seconds, which should be enough to capture its severity according to the same study.

The subjective test was run in a 21 -inch computer screen with HD resolution. Users sat in front of the screen at the distance they selected, and wore headphones for the audio. The sequences were randomly shown to the user by an application, and after each of them the user had to score it. The test application used Mplayer to display the content and to modify playback rate using the ScaleTempo open source plugin ${ }^{1}$.

The scoring of the sequences was done using Degradation Category Rating (DCR) [19]. As all the sequences included the reference speed at the beginning, the experiment can be considered a sort of double stimulus, and therefore DCR is a suitable scale for it. Besides, we wanted to capture whether the speed change was perceptible and, if it was, whether it was annoying or not, and this is perfectly captured by DCR, unlike other scales as Absolute Category Rating.

50 users (20 female, 30 male) took part on the experiment. All of them were university students (18-25 years old). Each participant rated the full combination of sources and rate variations $(15 \times 7)$.

\section{RESULTS}

Fig. 1 shows the Mean Opinion Score (MOS) for each of the values of the speed gain $G$. Differential MOS (DMOS) has also been computed using the hidden reference $(G=1)$ and the crushing algorithm for values higher than 5 defined in ITU-T P.910 [19].

\footnotetext{
${ }^{1}$ http://scaletempo.sourceforge.net
} 


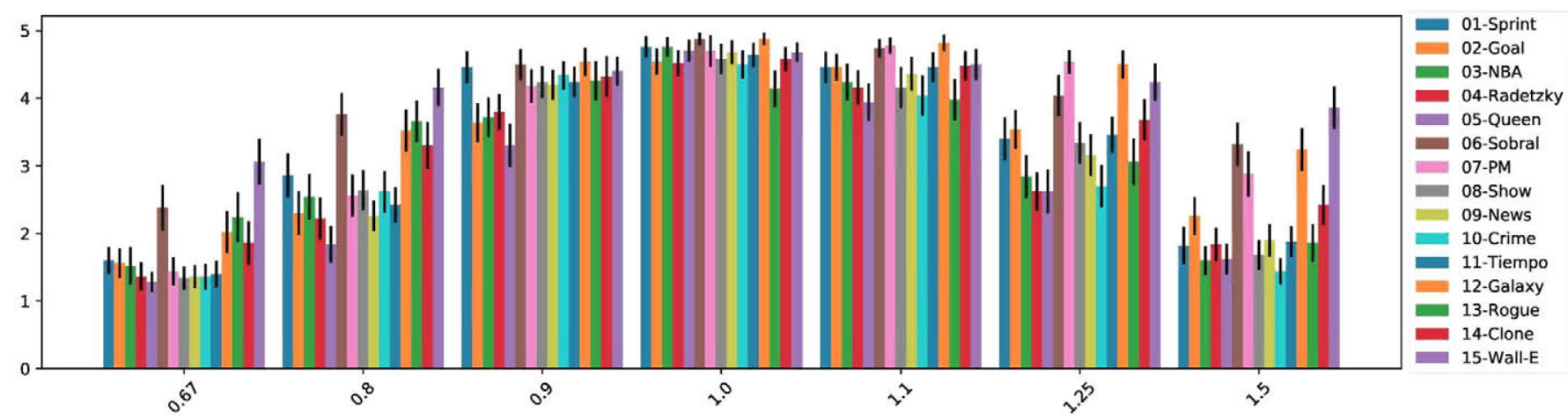

Fig. 2. MOS for each speed gain and source.

TABLE II

ANOVA TABLE FOR THE SCORES

\begin{tabular}{lrrrrrr}
\hline & SS & df & $F$ & $P(>F)$ & $\eta^{2}$ & $\omega^{2}$ \\
\hline $\mathrm{C}(G)$ & 5612 & 6 & 1120 & $0.000^{* * * *}$ & 0.50 & 0.50 \\
$\mathrm{C}(\mathrm{SRC})$ & 795 & 14 & 68 & $0.000^{* * * *}$ & 0.07 & 0.07 \\
$\mathrm{C}(G): \mathrm{C}(\mathrm{SRC})$ & 553 & 840 & 7.9 & $0.000^{* * * *}$ & 0.05 & 0.04 \\
Residual & 4298 & 5145 & - & - & - & - \\
$* * * p<0.001$ & & & & & &
\end{tabular}

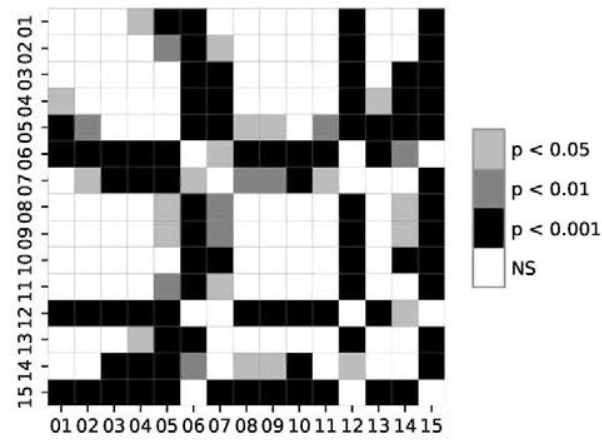

Fig. 3. Tukey's HSD post-hoc analysis of subject opinion scores with respect to source contents.

As there are 750 scores for each value of $G$, confidence intervals are very narrow. This shows two preliminary findings, all of them statistically significant under the conditions of our test: first, even relatively small distortions in the range of $10 \%$ are perceptible on average; and second, the perceived quality for any $G>1$ is higher than the quality of $1 / G$.

There are also strong variations among sources, as seen in Fig. 2. This means that the selection of the sources also has strong impact on the results. To test the effect of both factors (gain $G$ and source), a two-way ANOVA test was run (Table II). The effect of $G$ is statistically significant at $99.9 \%$ and very strong $\left(\eta^{2}=0.5\right)$. The effect of the sources, as well as the cross effect of source and gain, are also significant, though weaker. Tukey's Honest Significant Difference has been computed for the sources (Fig. 3), to show which sources behave significantly different from the others.
It is worth mentioning that parametric statistical analysis is being used in the assumption that there is a gaussian random process underneath the scoring. For robustness, Kruskal-Wallis tests have been run to validate the significance of $G$ and source in the scores, and a post-hoc Dunn test has been run to check the pairwise differences between sources. Results are qualitatively equivalent to the ones presented. A similar analysis, with equivalent results, has also been done for the differential opinion scores (i.e. DMOS instead of MOS).

It is noticeable that the MOS value for the hidden reference is quite below 5, meaning that some of the users voted that they perceived some degradation even if there was none. Fig. 4 (a) shows the distribution of scores for each $G$. There is a $27 \%$ of false positives: misdetection of speed variation on its absence. Similarly, scores of 5 for $G=0.67(2.5 \%)$ or $G=1.5(1.5 \%)$ can be considered false negatives, as distortions of such level are quite obvious.

The distribution of those kinds of "user errors" is heterogeneous among users, as seen in Fig. 4 (b). Some users can be considered fully reliable (not making any of those errors) while others raise up to a $80 \%$ of false positives in the hidden reference. We can define a reliability score Average Error Rate (AER) as

$$
\mathrm{AER}=\frac{\text { FalsePositives }(\%)+\text { FalseNegatives( } \%)}{2}
$$

For a first intuition on how this variability may affect the results, we have computed MOS for the good and bad sequences, meaning the seven sequences with higher and lower average MOS respectively. For each of those, we have also computed the MOS considering only the 20 most reliable users (which had an AER of $10 \%$ or less). As seen in Fig. 4 (c), the effect of user reliability is not negligible, but it is not critical either: about 0.25 MOS points in the worst case. However, the effect of content is higher: the deviation from average MOS is about 0.5 in each direction, meaning a difference of 1.0 between good and bad sources.

\section{USER MODEL}

To better understand the influence of user and source variability on the final opinion score, we have developed a 


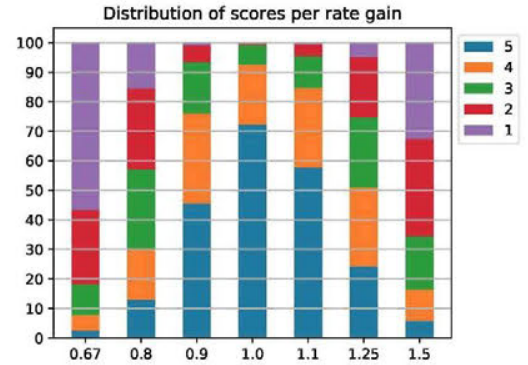

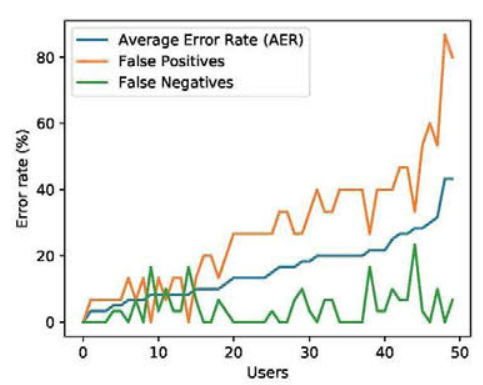

b

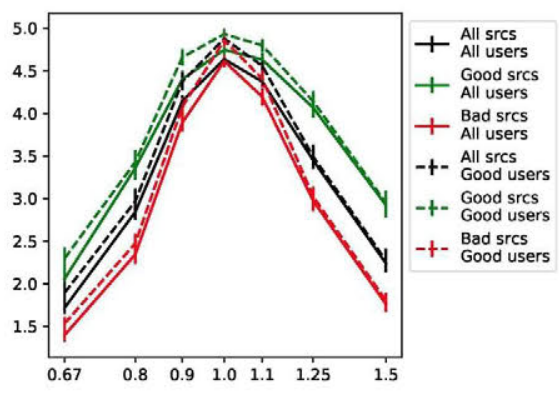

C

Fig. 4. a) Distribution of scores for each speed gain. b) Proportion of rate of false positives (for $G=1$ ) and false negatives (for $G \in\{0.67,1.5\}$ ) for each user, as well as the average between both (AER); users are sorted by AER. c) Variations of the scores for subsets of users and sources.
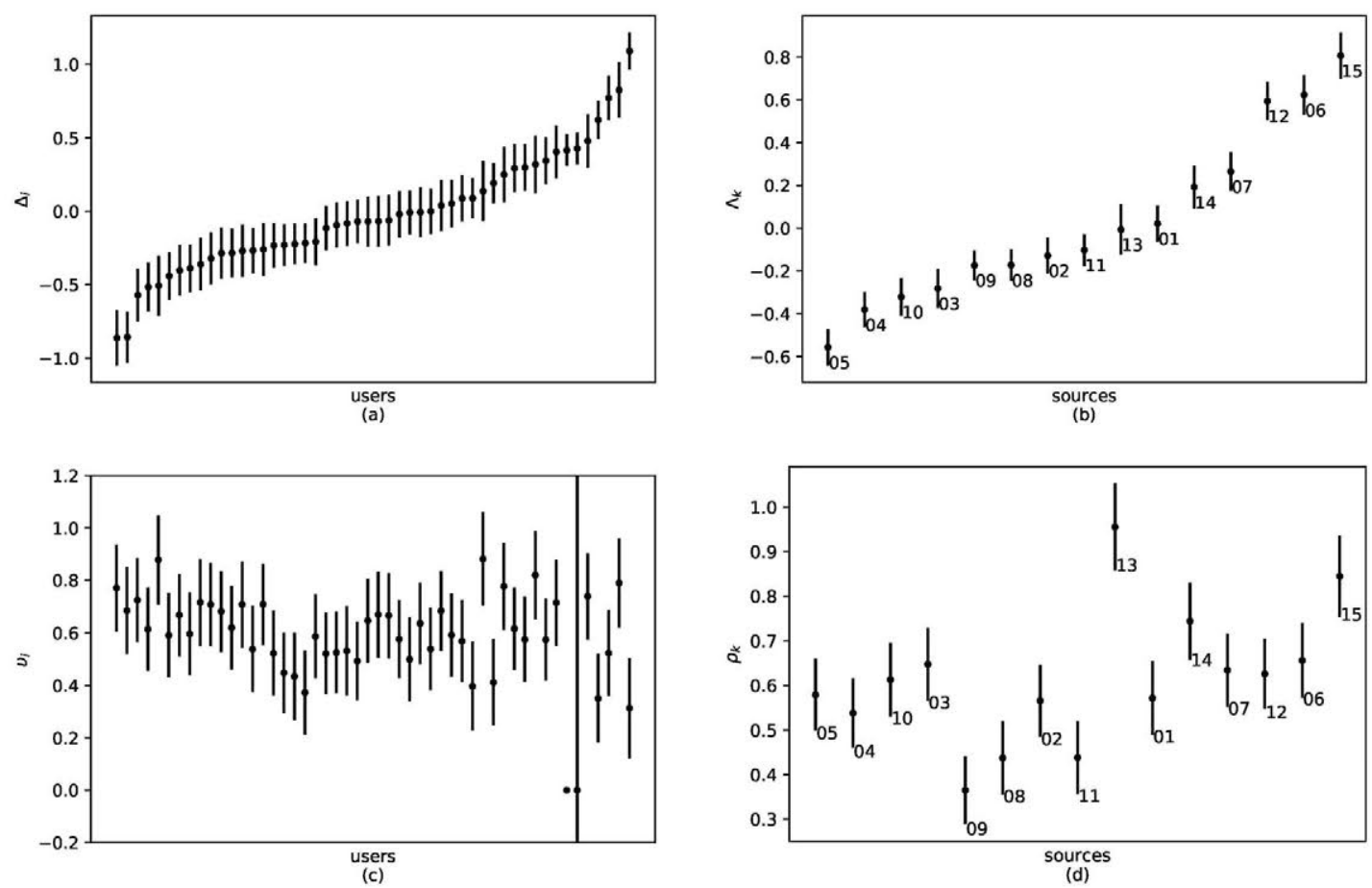

Fig. 5. User model. a) User bias $\Delta_{i}$, sorted from minimum to maximum. b) Content resilience $\Lambda_{k}$, sorted from minimum to maximum; source IDs are labeled. c) User variability $v_{i}$, sorted by $\Delta_{i}$. d) Content ambiguity $\rho_{k}$, sorted by $\Lambda_{k}$.

stochastic voting model based on the works of Janowski and Pinson [20] and $\mathrm{Li}$ and Bampis [21]. Our starting point is the model in [21]:

$$
U_{i, j}=\psi_{j}+\Delta_{i}+v_{i} X+\rho_{k(j)} Y
$$

where the evaluation of each user $i$ to each Processed Video Sequence (PVS) $j$ is modeled as a random variable $U_{i, j}$ depending on the true quality of the PVS $\psi_{j}$, the user bias and inconsistency $\left(\Delta_{i}, v_{i}\right)$, and the content ambiguity of each source $k\left(\rho_{k(j)}\right)$, and:

$$
X, Y \sim \mathcal{N}(0,1)
$$

Each PVS $j$ is actually the combination of a source $k$ and a rate gain $g$. To simplify the analysis, we assume that the contribution of each of those components to the PVS quality is additive and independent, in what can be seen as a first-order approximation to the actual (unknown) relation:

$$
\psi_{j}=\psi_{k, g} \approx \varphi_{g}+\Lambda_{k}
$$

where $\varphi_{g}$ is the quality value associated to the rate gain $g$ and $\Lambda_{k}$ is the content resilience to the rate variation. This allows us to write the scoring model as:

$$
U_{i, k, g}=\varphi_{g}+\Delta_{i}+v_{i} X+\Lambda_{k}+\rho_{k} Y
$$

Or, alternatively

$$
U_{i, k, g}=\varphi_{g}+\mathcal{X}_{i}+\mathcal{Y}_{k}
$$

where 
TABLE III

COMPARISON OF MODELS

\begin{tabular}{ccccccc}
\hline$G$ & MOS & DMOS & MLE & DMLE & {$[16]$} & {$[17]$} \\
\hline 0.67 & $1.72 \pm 0.07$ & $2.07 \pm 0.08$ & 1.67 & 2.04 & 4.51 & - \\
0.80 & $2.84 \pm 0.09$ & $3.16 \pm 0.09$ & 2.78 & 3.11 & 4.81 & - \\
0.90 & $4.14 \pm 0.07$ & $4.40 \pm 0.06$ & 4.17 & 4.39 & 4.94 & - \\
1.00 & $4.64 \pm 0.05$ & $5.00 \pm 0.00$ & 4.68 & 5.00 & 5.00 & 4.99 \\
1.10 & $4.37 \pm 0.06$ & $4.62 \pm 0.05$ & 4.41 & 4.60 & 5.00 & 4.45 \\
1.25 & $3.45 \pm 0.09$ & $3.75 \pm 0.08$ & 3.46 & 3.74 & 4.97 & 3.74 \\
1.50 & $2.24 \pm 0.08$ & $2.59 \pm 0.09$ & 2.22 & 2.58 & 4.79 & 2.80 \\
\hline
\end{tabular}

$\pm C I$ means $95 \%$ Confidence Interval. $C I$ for MLE and DMLE is $0.06 \forall G$

$$
\begin{aligned}
& \mathcal{X}_{i} \sim \mathcal{N}\left(\Delta_{i}, v_{i}\right) \\
& \mathcal{Y}_{k} \sim \mathcal{N}\left(\Lambda_{k}, \rho_{k}\right)
\end{aligned}
$$

To find the values of the model parameters, we have used Maximum Likelihood Estimation (MLE) with the Belief Propagation algorithm proposed in [21] and the following loglikelihood function:

$$
\begin{aligned}
L(\theta) & =\log (P)\left(\left\{u_{i, k, h}\right\} \mid \theta\right) \\
& =\log (P)\left(\left\{u_{i, k, h}\right\} \mid\left\{\varphi_{g}\right\},\left\{\Lambda_{k}\right\},\left\{\rho_{k}\right\},\left\{\Delta_{i}\right\},\left\{v_{i}\right\}\right) \\
& =\sum_{i, k, g}-\frac{1}{2} \log \left(\rho_{k}^{2}+v_{i}^{2}\right)-\frac{1}{2} \frac{\left(u_{i, k, g}-\varphi_{g}-\Lambda_{k}-\Delta_{i}\right)^{2}}{\rho_{k}^{2}+v_{i}^{2}}
\end{aligned}
$$

The results for the MLE $\varphi_{g}$ are identical to the MOS, within confidence intervals (see Table III). The rest of the parameters are displayed in Fig. 5. Both $\Delta_{k}$ and $\Lambda_{k}$ follow normal distributions, according to a D'Agostino and Pearson's test, with:

$$
\begin{aligned}
& \Delta_{k} \sim \mathcal{N}(-0.02,0.40) \\
& \Lambda_{k} \sim \mathcal{N}(0.03,0.38) \\
& \text { V. Discussion }
\end{aligned}
$$

\section{A. Average quality}

Table III shows the results of our tests, including confidence intervals. DMLE is the maximum likelihood estimation of the differential scores used to compute DMOS. The models from Rainer and Timmerer [16] and $\mathrm{Mu}$ et al. [17] (for AMP duration of 6 seconds) are also displayed.

Our results of DMOS/DMSE are consistent with [17], which is the only work, to our knowledge, which has performed subjective tests with realistic content for video streaming. The model of [16] clearly overestimates the quality, as it has been developed from a single animation content, with high resilience to speed variations. Also, the assumption that $25 \%$ variations are not perceptible and $50 \%$ variations are acceptable [8] is too optimistic: a $10 \%$ of maximum variation is much safer in terms of not being noticeable or, at least, annoying. It
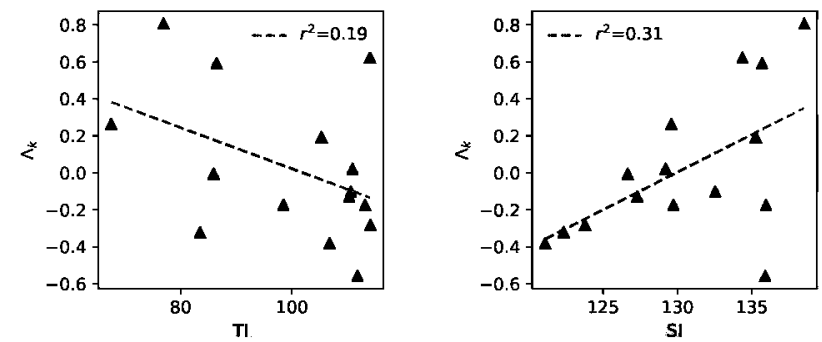

Fig. 6. Scatter plots of $\Lambda_{k}$ vs. TI (left) and SI (right), including linear fit and coefficient of determination $r^{2}$.

is also worth noting that even $\pm 10 \%$ variations are statistically significantly different from the reference. Additionally, most of the cost models use symetrical degradations for speed decrease and increase (e.g. [10], [13], [14]), neglecting the fact that the former has higher impact in quality than the latter.

From our results, we can propose a simple piecewise linear fit as cost model:

$$
\operatorname{DMOS}(G)= \begin{cases}-4.1+9.1 G, & \text { for } G \leq 1 \\ 9.9-4.9 G, & \text { for } G>1\end{cases}
$$

However, we have provided the ground truth values in Table III so that it is easy for anyone to use smoother interpolators, such as higher order polynomials, if required.

\section{B. Impact of source}

We have shown that there is significant variation in the results from content to content. Differences in content resilience to AMP $\left(\Lambda_{k}\right)$ across sources (Fig. 5) are consistent with the post-hoc analysis of the user scores (Fig.3), which, together with the equality of MLE and MOS scores, supports the validity of our user model parameter computation. Additionally, content ambiguity $\rho_{k}$ is higher that the one reported for subjective analysis of coding artifacts [21].

Unfortunately, there is no trivial relationship between source influence and simple low-level features of the content. Fig. 6 shows the low correlation between the content resilience $\Lambda_{k}$ and basic video statistics such as Temporal Information and Spatial Information, as defined in [19]. Using motion information as in [15] or [16] does not seem sufficient to characterize the content.

Qualitatively, the best responses have been obtained for animation sources $(12,14,15)$ and action (13-15). Action sequences are, on the other hand, more difficult to evaluate (higher $\rho_{k}$ ). Rhythmic music $(04,05)$ is strongly affected by AMP, unlike melodic music (06). Further research should be done to describe all these properties quantitatively.

\section{Impact of user}

User bias and variability behaves similarly to other types of subjective assessment, although the distribution of bias is wider $(\sigma=0.40)$ than what has been reported for evaluation of video capture and compression artifacts $(\sigma=0.34$ [20]). This may have relation with the fact that users are less familiar to 

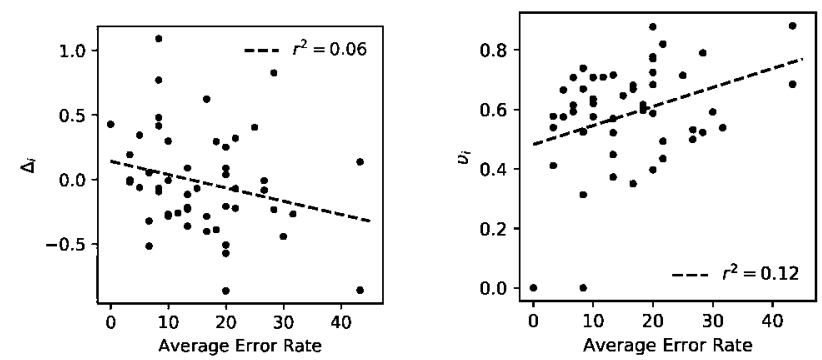

Fig. 7. Scatter plots of AER vs. $\Delta_{i}$ (left) and $v_{i}$ (right), including linear fit and coefficient of determination $r^{2}$.

playout speed variation artifacts, and therefore their subjective sensitivity to them is less homogeneous.

As in other studies with hidden reference, the scoring of such reference was not perfect [16]. This has led us to define a metric of user Average Error Rate (AER), considering the situations where we can argue that the user opinion is objectively incorrect. Taking only the opinions of users with low AER, subjective MOS increases in about 0.25 points. However, the correlation between AER and user bias or inconsistency is extremely low (Fig. 7), which suggests that the ability of the users to detect specific impairments may be independent to their general bias or variability on their opinions. Further research would be needed to validate this hypothesis.

\section{CONCLUSIONS}

The study of the subjective impact of adaptive media playout (AMP) had been very limited so far. We have performed an extensive subjective test in terms of sources and users, which can provide some practical guidelines for AMP implementations in the future.

As a first conclusion, we have found that most previous studies underestimated the subjective impact of AMP. We have proposed a $10 \%$ of playout rate variation that can be used as "rule of thumb" for simple implementations, and we have also provided a cost function for more complex ones.

We have also evaluated the impact of individual sources and user variability on the building of the opinion scores. We have proposed a user model to analyze the influence of source, subject, and speed gain on the quality scores. The results provide very useful insights on the source content resilience to the impairment, and we believe that such analysis might be useful in other kinds of degradation, such as compression.

There is a significant variation on the scores depending on the content resilience to AMP. Animation contents or contents without people speaking have highest resilience, while contents with strong periodicity (e.g. rhythmic music) have the lowest. Further research should be done to quantify this property with objective metrics.

\section{ACKNOWLEDGEMENT}

Authors wish to thank Alejandra Imaz and Mateo Cámara for their help with the subjective assesment tests.

\section{REFERENCES}

[1] C.-Y. Hsu, A. Ortega, and M. Khansari, "Rate control for robust video transmission over burst-error wireless channels," IEEE J. Sel. Areas Commun., vol. 17, no. 5, pp. 756-773, May 1999

[2] M.-N. Garcia, F. De Simone, S. Tavakoli, N. Staelens, S. Egger, K. Brunnström, and A. Raake, "Quality of experience and HTTP adaptive streaming: A review of subjective studies," in Proc. 6th Int. Workshop Quality of Multimedia Experience (QoMEX), 2014.

[3] R. Mekuria, P. Cesar, and D. Bulterman, "Digital TV: the effect of delay when watching football," in Proc. 10th European Conf. Interactive TV and Video, 2012, pp. 71-74.

[4] W. J. Kooij, H. M. Stokking, R. van Brandenburg, and P.-T. de Boer, "Playout delay of TV signals: measurement system design, validation and results," in Proc. ACM Int. Conf. Interactive Experiences for TV and Online Video, 2014, pp. 23-30.

[5] M. Montagud, F. Boronat, H. Stokking, and R. van Brandenburg, "Inter-destination multimedia synchronization: schemes, use cases and standardization," Multimedia Systems, vol. 18, no. 6, pp. 459-482, 2012.

[6] E. Steinbach, N. Farber, and B. Girod, "Adaptive playout for low latency video streaming," in Proc. IEEE Int. Conf. Image Processing (ICIP), 2001, pp. 962-965.

[7] Y. J. Liang, N. Farber, and B. Girod, "Adaptive playout scheduling using time-scale modification in packet voice communications," in Proc. IEEE Int. Conf. Acoustics, Speech, and Signal Processing (ICASSP), vol. 3, 2001, pp. 1445-1448.

[8] M. Kalman, E. Steinbach, and B. Girod, "Adaptive media playout for low-delay video streaming over error-prone channels," IEEE Trans. Circuits Syst. Video Technol., vol. 14, no. 6, pp. 841-851, 2004.

[9] Y.-F. Su, Y.-H. Yang, M.-T. Lu, and H. H. Chen, "Smooth control of adaptive media playout for video streaming," IEEE Trans. Multimedia, vol. 11, no. 7, pp. 1331-1339, 2009.

[10] J. Wang, W. Lei, P. Xu, J. Sun, and Z. Guo, "Adaptive media playout buffer management for latency optimization of mobile live streaming," in Proc. IEEE Int. Conf. on Multimedia Expo Workshops (ICMEW), July 2017, pp. 369-374.

[11] T. Ozcelebi, A. M. Tekalp, and M. R. Civanlar, "Delay-distortion optimization for content-adaptive video streaming," IEEE Trans. Multimedia, vol. 9, no. 4, pp. 826-836, 2007.

[12] B. Rainer and C. Timmerer, "Self-organized inter-destination multimedia synchronization for adaptive media streaming," in Proc. 22nd ACM Int. Conf. on Multimedia, 2014, pp. 327-336.

[13] M. Montagud, F. Boronat, and H. Stokking, "Design and simulation of a distributed control scheme for inter-destination media synchronization," in Proc. IEEE 27th Int. Conf. Advanced Information Networking and Applications (AINA), 2013, pp. 937-944.

[14] M. Kalman, E. Steinbach, and B. Girod, "RD optimized media streaming enhanced with adaptive media playout," in Proc. IEEE Int. Conf. Multimedia and Expo (ICME), vol. I, 2002, pp. 869-872.

[15] Y. Li, A. Markopoulou, J. Apostolopoulos, and N. Bambos, "Contentaware playout and packet scheduling for video streaming over wireless links," IEEE Trans. Multimedia, vol. 10, no. 5, pp. 885-895, 2008.

[16] B. Rainer and C. Timmerer, "A quality of experience model for adaptive media playout," in Proc. 6th Int. Workshop Quality of Multimedia Experience (QOMEX), 2014.

[17] M. Mu, L. Fawcett, J. Bird, J. Jellicoe, S. Simpson, H. Stokking, and N. Race, "Closing the gap: human factors in cross-device media synchronization," IEEE J. Sel. Topics Signal Process., vol. 11, no. 1, pp. 180-195, 2017.

[18] H. Hoffmann, T. Itagaki, D. Wood, and A. Bock, "Studies on the bit rate requirements for a HDTV format with $1920 \times 1080$ pixel resolution, progressive scanning at $50 \mathrm{hz}$ frame rate targeting large flat panel displays," IEEE Trans. Broadcast., vol. 52, no. 4, pp. 420-434, 2006.

[19] "ITU-T P.910. Subjective video quality assessment methods for multimedia applications," International Telecommunication Union, Recommendation, Apr. 2008.

[20] L. Janowski and M. Pinson, "The accuracy of subjects in a quality experiment: A theoretical subject model," IEEE Trans. Multimedia, vol. 17, no. 12, pp. $2210-2224,2015$.

[21] Z. Li and C. G. Bampis, "Recover subjective quality scores from noisy measurements," in Data Compression Conference $(D C C), 2017$. IEEE, 2017, pp. 52-61. 